\title{
MAGNETIC BRIGHT POINTS IN THE QUIET SUN
}

\author{
J. Sánchez Almeida ${ }^{1,2}$, J. A. Bonet 1,2, B. Viticchié ${ }^{3,4}$, And D. Del Moro ${ }^{4}$ \\ ${ }^{1}$ Instituto de Astrofísica de Canarias, E-38205 La Laguna, Tenerife, Spain; jos@iac.es, jab@iac.es \\ 2 Departamento de Astrofísica, Universidad de La Laguna, E- 38071 La Laguna, Tenerife, Spain \\ ${ }^{3}$ ESA/ESTEC RSSD, Keplerlaan 1, 2200 AG Noordwijk, The Netherlands; Bartolomeo.Viticchie@esa.int \\ ${ }^{4}$ Dipartimento di Fisica, Università degli Studi di Roma “Tor Vergata," I-00133 Rome, Italy; delmoro@roma2.infn.it \\ Received 2010 March 5; accepted 2010 April 9; published 2010 April 28
}

\begin{abstract}
We present a visual determination of the number of bright points (BPs) existing in the quiet Sun, which are structures though to trace intense $\mathrm{kG}$ magnetic concentrations. The measurement is based on a $0^{\prime \prime} 1$ angular resolution G-band movie obtained with the Swedish Solar Telescope at the solar disk center. We find $0.97 \mathrm{BPs} \mathrm{Mm}^{-2}$, which is a factor 3 larger than any previous estimate. It corresponds to $1.2 \mathrm{BPs}$ per solar granule. Depending on the details of the segmentation, the BPs cover between $0.9 \%$ and $2.2 \%$ of the solar surface. Assuming their field strength to be $1.5 \mathrm{kG}$, the detected BPs contribute to the solar magnetic flux with an unsigned flux density between $13 \mathrm{G}$ and $33 \mathrm{G}$. If network and inter-network regions are counted separately, they contain $2.2 \mathrm{BPs} \mathrm{Mm}^{-2}$ and $0.85 \mathrm{BPs} \mathrm{Mm}^{-2}$, respectively.
\end{abstract}

Key words: Sun: activity - Sun: dynamo - Sun: granulation - Sun: photosphere - Sun: surface magnetism

\section{MOTIVATION}

Our understanding of the quiet-Sun magnetic fields has turned over during the last decade. Traditional magnetograms showed magnetic signals only at the network boundaries, occupying only a small fraction of the solar surface, and mainly produced by spatially unresolved $\mathrm{kG}$ magnetic concentrations (e.g., Beckers 1977; Solanki 1993). Nowadays, magnetic signals are detected almost everywhere (e.g., Domínguez Cerdeña et al. 2003; Harvey et al. 2007; Lites et al. 2008), and there is no doubt on the presence of a volume-filling component of $\mathrm{dG}$ and hG fields as inferred from the Hanle depolarization signals (e.g., Faurobert-Scholl 1993; Trujillo Bueno et al. 2004). The intranetwork (IN) magnetic fields have passed from hardly delectable signals in long-integration low spatial resolution magnetograms (e.g., Martin 1988; Wang et al. 1995) to ubiquitous features (e.g., Lin \& Rimmele 1999; Sánchez Almeida \& Lites 2000). The abundance of new magnetic structures makes them potentially important to understand the global magnetic properties of the Sun (Sánchez Almeida 2004; Trujillo Bueno et al. 2004), and also makes it unlikely that the quiet-Sun magnetism results from the decay of active regions (e.g., Sánchez Almeida et al. 2003). Since the quiet-Sun magnetic fields do not seem to be debris from active regions, they are not automatically generated by the dynamo responsible for the solar cycle. A different production mechanism seems to be at work, probably an efficient turbulent dynamo driven by granular convection (Petrovay \& Szakaly 1993; Cattaneo 1999), a hypothesis corroborated by the latest numerical simulations of granular magneto-convection (Vögler \& Schüssler 2007; Pietarila Graham et al. 2010). The sensitivity of the current polarimeters allow us to detect weak linear polarization signals produced by the transverse component of the magnetic fields (Harvey et al. 2007; Lites et al. 2008), and to find out that they often emerge as short loops popping up from the sub-photosphere (Centeno et al. 2007; Martínez González \& Bellot Rubio 2009). In agreement with the turbulent dynamo scenario, quiet-Sun magnetic fields come with strengths in the full range from basically zero to $2 \mathrm{kG}$ (Sánchez Almeida \& Lites 2000; Domínguez Cerdeña et al. 2006). Even if they only fill a small fraction of the quiet photosphere, the part having strong $\mathrm{kG}$ fields may be particularly important from a physical point of view. Magnetic flux and magnetic energy scale as powers of the field strength, therefore, depending on their (uncertain) area coverage, kGs may surpass the contribution of the more common but weaker fields (Sánchez Almeida 2004). Moreover, buoyancy makes kG concentrations vertical (e.g., Schüssler 1986) and so, they can provide a mechanical connection between the photosphere and the upper atmosphere (e.g., van Ballegooijen et al. 1998; Sánchez Almeida et al. 2007). Theoretical studies support the traditionally ignored but potentially important influence of the quiet-Sun photosphere on the chromosphere, transition region, and corona (e.g., Schrijver \& Title 2003; Goodman 2004; Jendersie \& Peter 2006). Despite all these (and other) significant advances, our understanding of the quiet-Sun magnetism is still incomplete. Each observational improvement reveals more magnetic structures, in a process showing no sign of having converged yet.

As a part of this movement to characterize the properties of the quiet-Sun magnetism, we discovered the ubiquitous presence of bright points (BPs) in the intergranular lanes of the quiet Sun (Sánchez Almeida et al. 2004). The plasma in the lanes is cold and therefore dark, but the BPs look bright because they trace intense kG magnetic concentrations (Spruit 1977). The magnetic field provides most of the pressure required to maintain the structure in mechanical balance, which reduces the gas pressure, the density and, thus, the opacity. The $\mathrm{kG}$ concentrations resemble holes carved on the solar surface which allow us to peep up into the sub-photosphere, where deeper usually means hotter and brighter. This picture is confirmed by realistic numerical simulations (e.g., Carlsson et al. 2004; Keller et al. 2004), and thus BPs are regarded as proxies for $\mathrm{kG}$ magnetic concentrations. The presence of BPs everywhere on the quiet Sun has been verified in several works, e.g., de Wijn et al. (2005, 2008); Bovelet \& Wiehr (2008); Sánchez Almeida et al. (2007). However, none of these works find as many BPs as Sánchez Almeida et al. (2004; 0.3 BPs $\mathrm{Mm}^{-2}$ ). This is partly due to the angular resolution of the images, taken with small telescopes, and also due to the use of automatic algorithms for BP identification which can hardly detect the faintest BPs. In an effort to calibrate with visual detection one of these methods (Bovelet \& Wiehr 2001, 2007), we repeated the tedious but otherwise accurate analysis carried out by Sánchez Almeida et al. (2004). This time we use a G-band image of much better quality. The result of such detailed analysis is surprising, and 


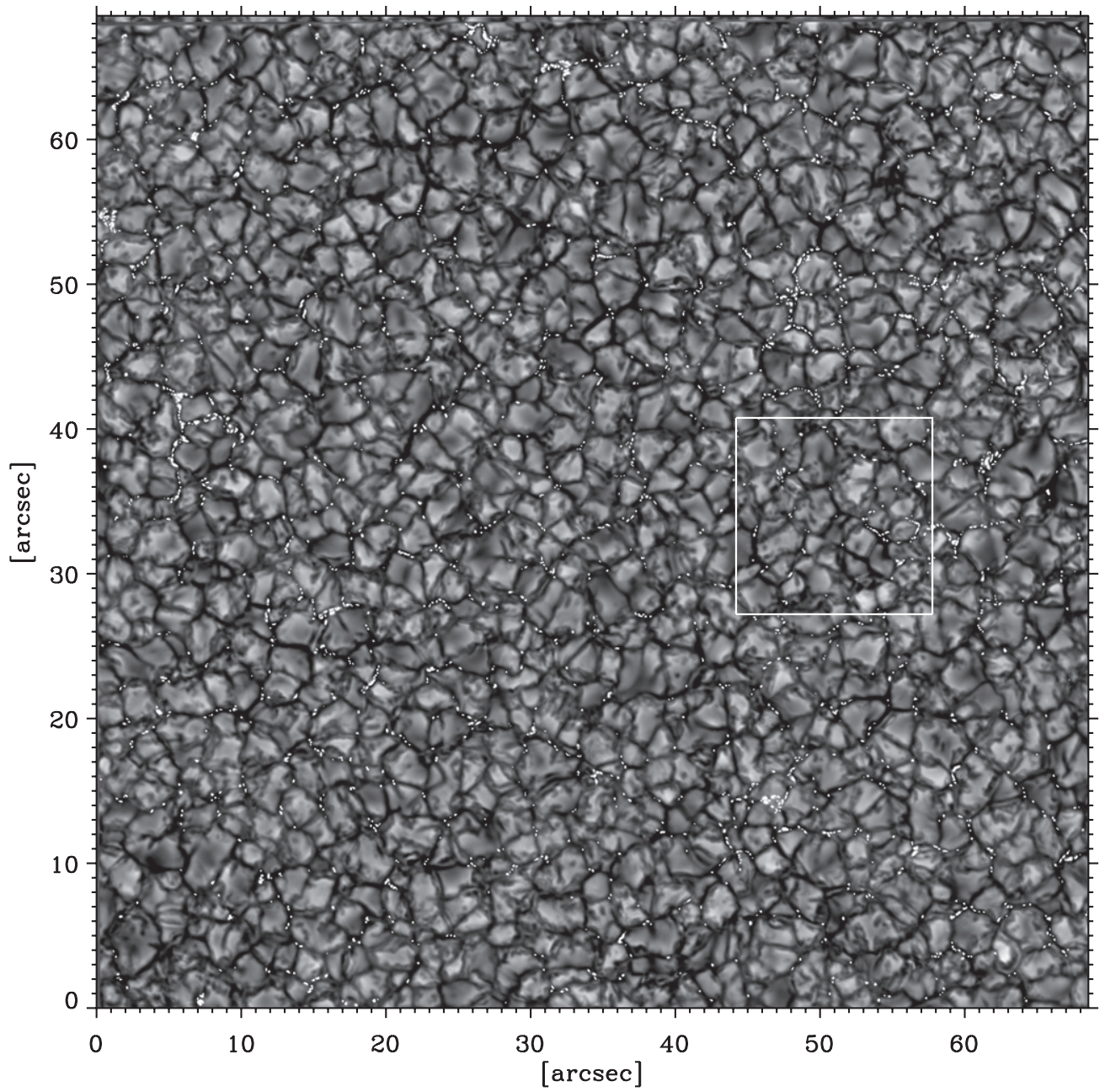

Figure 1. G-band image enhanced at the position of the selected BPs. BPs are found throughout, with a number density of $0.97 \mathrm{BPs}_{\mathrm{Mm}}^{-2}$ or, equivalently, of 1.2 BPs per granule. The square box outlines the region blown up in Figure 2.

we report it here. We find three times more G-band BPs than in the original study and, therefore, far more than the numbers reported in all the previous studies cited above. Our density implies that the quiet Sun has more BPs than granules, and at least $1 \%$ of the quiet solar surface is covered by BPs.

\section{OBSERVATION AND ANALYSIS PROCEDURE}

Figure 1 shows the image selected for in-depth analysis. It is the best in a series of quiet-Sun disk center images taken with the Swedish Solar Telescope (SST; Scharmer et al. 2002) in the G band (a $10.8 \AA$ Aide filter centered at $4305.6 \AA$ ). The $68.5 \times 68^{\prime \prime} .5$ field of view (FOV) was observed on 2007 September 29. The series was restored using multi-frame blind deconvolution (van Noort et al. 2005) to have an angular resolution close to the diffraction limit of the SST at the working wavelength $\left(\simeq 0{ }^{\prime \prime} 1\right)$. Each snapshot results from combining 125 images, rendering a mean cadence of one snapshot every $15 \mathrm{~s}$. The data set includes simultaneous $\mathrm{Ca} \mathrm{H}$ line-core images, which we use to determine network boundaries. Further details are given by Bonet et al. (2008).

The BPs were selected in a way similar to that described by Sánchez Almeida et al. (2004). The reference image was segmented into locally bright features and background using the algorithm by Strous (1994). We flick on the computer screen the segmented image and the true image, selecting as BPs bright structures coinciding with one of the segmented patches. We first pinpoint obvious BPs in the intergranular lanes of the best image. In case of doubt, we play back and forth the animation with snapshots around it. We then select as BPs in our image those that along the time sequence show up as clean BPs (see the example in Figure 2(d)). Doubtful identifications were discarded. Moreover, we also include BPs that were conspicuous before or after, but which remain as faint brightenings in the reference snapshot because of evolution. The procedure was repeated using two different saturation levels for the true image. The low contrast (between 0.8 and 1.5, in units of the mean quiet-Sun intensity) was used to select the brightest BPs, whereas the high contrast (between 0.7 and 1.1) was used for dimmer BPs. Figures 2(a) and (b) contain a small part of the full FOV shown with these two different contrasts. (A box outlines the subfield in Figure 1.)

The disk center was not fully devoid of magnetic activity during the observation. There was a small nearby active region (AR NOAO $10971^{5}$ ) that the telescope pointing avoided. We discard the influence of this AR on the observed region with the following arguments: (1) a simultaneous Hinode SOT/NB Na I $5896 \AA$ A magnetogram including the outskirts of the AR and the SST FOV shows that the two of them are well separated. (2) The average unsigned magnetic flux density in our FOV (29 G) is within the low range of values of the signals in a magnetogram of the quiet Sun taken under the same conditions ten days later,

5 It never developed fully, producing only a few pores during its peak activity. 

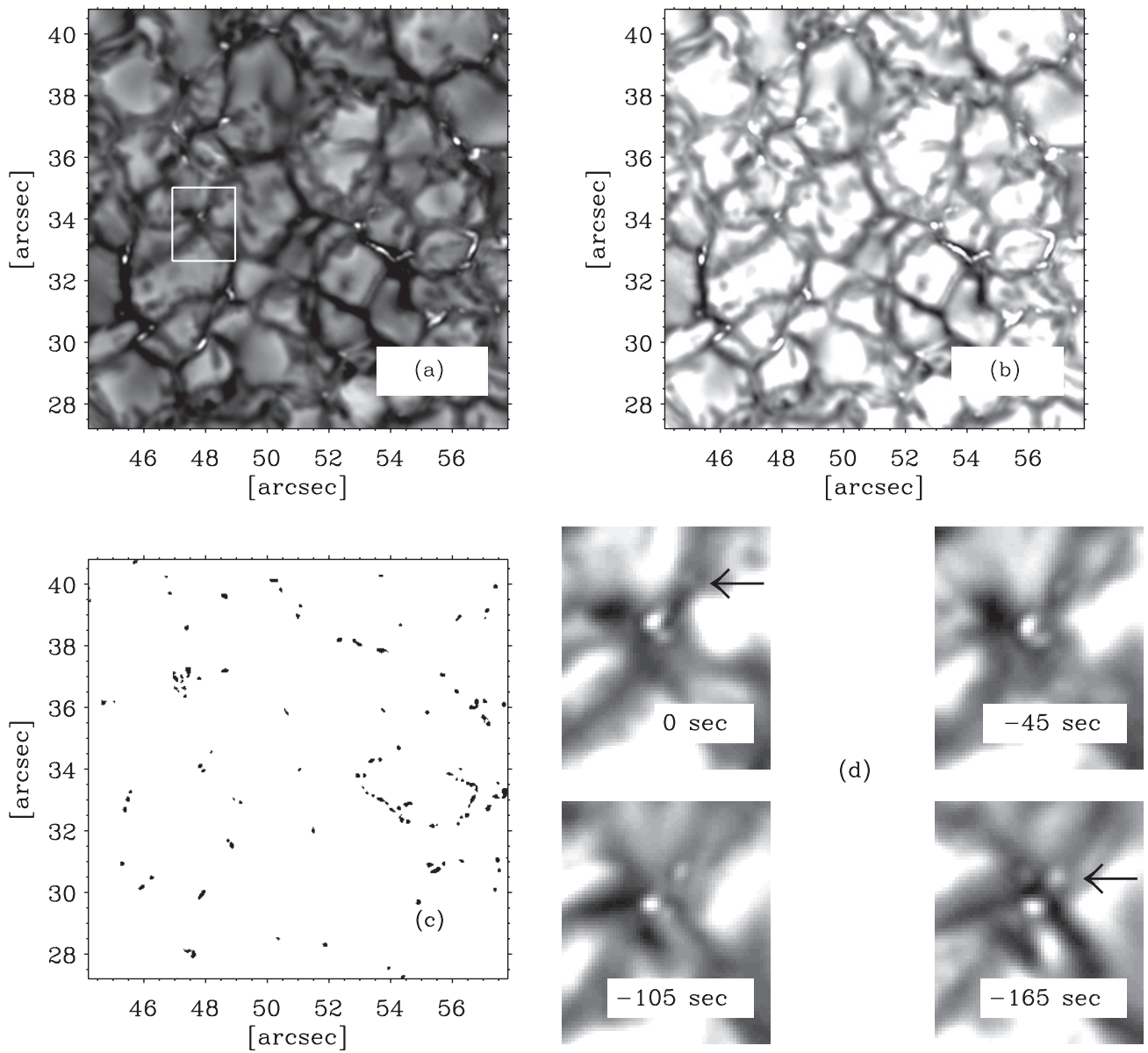

(d)

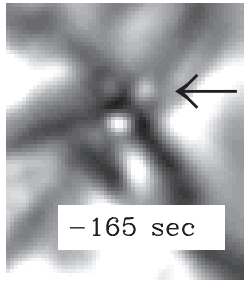

Figure 2. Illustration of the visual selection procedure. (a) Low contrast image of a small subfield of the FOV (see the box in Figure 1). It is used to select the brightest G-band BPs. (b) Same image as (a) shown with high contrast. Used to identify fainter BPs. (c) Binary map with the selected structures. (d) Time series (see the insets) illustrating how an uncertain BP in the reference image (time $0 \mathrm{~s}$, the arrow) was actually a clear and conspicuous BP a few minutes before (time $-165 \mathrm{~s}$, the arrow). The subfield shown in the time series is marked as a box in (a)

when no AR was present. This reference magnetogram yields a signal of $34 \pm 5 \mathrm{G}$, with the error bar representing the standard deviation among the signals in randomly chosen subfields with the size of the SST FOV.

\section{RESULTS}

Figure 1 shows the result of our selection. The original Gband image has been enhanced in those segmented structures identified as BPs. One can recognize super-granulation and meso-granulation scales, although BPs are found throughout the FOV. We identify $2380 \mathrm{BPs}$, which correspond to 0.97 BPs $\mathrm{Mm}^{-2}$. In the segmentation employed for BP selection, these BPs cover $0.89 \%$ of the surface (the so-called filling factor $f$ ). However, the filling factor is an uncertain parameter. (The number density of BPs is far more robust.) If the segmentation algorithm is used dilating the kernel by 1 pixel (roughly speaking, increasing the size of the individual BPs by 1 pixel), then the surface coverage becomes $2.2 \%$. This other segmentation is as good as the original one as judged from visual inspection. The segmentation algorithm underestimates the area covered by the large BPs. They are split into several individual objects, and the empty space left between them does not contribute to $f$ (compare the bright continuous filaments in Figure 2(b) with the corresponding broken segmentation in Figure 2(c)). The splitting of one large object into several small pieces also enlarges our BP number density. However, this bias does not have a big impact because large objects are mostly in the network, and when network and IN are treated separately, the resulting number densities turn out to be very similar (see below).

We have tried to separate the contribution of network and IN. This separation is based on the $\mathrm{Ca} \mathrm{H}$ images taken simultaneously with the G-band images. They are smoothed with a 4" kernel, with the bright patches above a threshold representing the network. The threshold was tuned to get a $10 \%$ area coverage, which is the kind of network surface coverage during solar minimum (e.g., Foukal \& Milano 2001; Sánchez Almeida 2004). Number densities and filling factors were separately recomputed for the pixels inside and outside the network thus defined. The number density of BPs and the filling factor of the IN are slightly smaller than the ones for the full FOV, but not so much. One gets $0.85 \mathrm{BPs} \mathrm{Mm}^{-2}$ and $f=0.77 \%$ when the full FOV rendered $0.97 \mathrm{BPs} \mathrm{Mm}^{-2}$ and $0.89 \%$, respectively. This small difference is due to the fact that BPs are found throughout, rather than being concentrated in the network. The density in the network patches is $2.2 \mathrm{BPs} \mathrm{Mm}^{-2}$, whereas $f=2.2 \%$.

As we explain in Section 1, our work was originally motivated to calibrate the automatic procedure by Bovelet \& Wiehr (2001, 2007). A detailed comparison goes beyond the scope of this Letter, but the algorithm systematically underestimates the number of BPs, so that it yields $0.18 \mathrm{BPs} \mathrm{Mm}^{-2}$. The patches associated to these BPs are substantially larger than those inferred here, so that $f$ remains $\simeq 2 \%$. 


\section{DISCUSSION}

According to the current paradigm, the BPs trace intense magnetic concentrations; so, our observation constrains the fraction of quiet-Sun plasma having kGs. Our measurement should be regarded as a lower limit since we only include secure BPs (Section 2), the area of large BPs is underestimated (Section 3), and diverse arguments suggest that some kGs are not bright (e.g., Sánchez Almeida et al. 2001; Vögler et al. 2005; Beck et al. 2007). Moreover, we miss BPs since the detectability of the small ones critically depends on the resolution (e.g., Title \& Berger 1996), and quiet-Sun BPs are often at the resolution of the observation (e.g., Sánchez Almeida et al. 2004; Bovelet $\&$ Wiehr 2008). In fact, differences of angular resolution partly explain the lower number density of BPs previously reported in the literature (Section 1). Another part of the difference is due to the use of automatic algorithms based on single frames to detect the BPs, which are still unable to identify the smallest and dimmest BPs in the images (Section 3).

Even a $1 \%$ filling factor in the form of $\mathrm{kG}$ fields represents a big challenge. They are not yet produced by the current turbulent solar dynamo simulations (Vögler \& Schüssler 2007), and they are overlooked in recent studies of the quiet-Sun magnetic field strength distribution based on Stokes spectro-polarimetry (e.g., Orozco Suárez et al. 2007; Asensio Ramos 2009). Assuming that typical BPs have $1.5 \mathrm{kG}$ field strengths, they contribute to the solar magnetic fields with an unsigned flux density between $13 \mathrm{G}$ and $33 \mathrm{G}$ depending on whether we adopt $0.9 \%$ or $2.2 \%$ for their filling factors. However, Orozco Suárez et al. (2007) report only $9.5 \mathrm{G}$, with field strengths dominated by hG rather than $\mathrm{kG}$ fields. The solution to this double puzzle may also have to do with insufficient spatial resolution. On the one hand, the amount of $\mathrm{kG}$ fields created by magneto-convection simulations increases with increasing spatial resolution (e.g., Bushby et al. 2008). On the other hand, the standard procedure of interpreting Stokes profiles does not acknowledge the existence of unresolved structuring. When it is acknowledged, then $\mathrm{kG}$ fields with filling factors compatible with the limits posed here are found (Viticchié et al. 2010).

Figure 1 shows BPs throughout. Their abundance can be emphasized by comparison with the number of granules. There are some 0.82 granules $\mathrm{Mm}^{-2}$ (e.g., Muller 2000), which implies that the quiet Sun has more BPs than granules, i.e., our image has 1.2 BPs per granule. Yet another revealing reference to compare with is the largest sunspot-group ever photographed on the Sun. It seems to correspond to a complex active region observed on 1947 April 7, which occupied $4.3 \times 10^{3}$ millionths of solar hemisphere (Hoge 1947). This record-breaking large sunspot yielded only $f \simeq 0.2 \%$, i.e., one would need several ARs like this one to carry the same unsigned flux as the quiet-Sun BPs have at any time if they uniformly cover the solar surface.

The SST is operated in the Spanish Observatorio del Roque de los Muchachos by the Institute for Solar Physics of the Royal Swedish Academy of Sciences. Hinode is a Japanese mission developed and launched by ISAS/JAXA, with NAOJ, NASA (US), and STFC (UK) as partners. We thank A. de Vicente for his support on the Condor ${ }^{6}$ workload management system used during image restoration. The work has been partly funded by the Spanish Ministries of Education, and of Science and Innovation (projects AYA2007-66502, AYA2007-63881, and ESP2006-

\footnotetext{
6 http://www.cs.wisc.edu/condor/
}

13030-C06-04), and by the EC (SOLAIRE Network-MTRNCT-2006-035484).

Facilities: SST, Hinode (SOT/NB)

\section{REFERENCES}

Asensio Ramos, A. 2009, ApJ, 701, 1032

Beck, C., Bellot Rubio, L. R., Schlichenmaier, R., \& Sütterlin, P. 2007, A\&A, 472, 607

Beckers, J. M. 1977, in Illustrated Glossary for Solar and Solar-Terrestrial Physics, ed. A. Bruzek \& C. J. Durrant (Dordrecht: Reidel), 21

Bonet, J. A., Márquez, I., Sánchez Almeida, J., Cabello, I., \& Domingo, V. 2008, ApJ, 687, L131

Bovelet, B., \& Wiehr, E. 2001, Sol. Phys., 201, 13

Bovelet, B., \& Wiehr, E. 2007, Sol. Phys., 243, 121

Bovelet, B., \& Wiehr, E. 2008, A\&A, 488, 1101

Bushby, P. J., Houghton, S. M., Proctor, M. R. E., \& Weiss, N. O. 2008, MNRAS, 387,698

Carlsson, M., Stein, R. F., Nordlund, Å., \& Scharmer, G. B. 2004, ApJ, 610, L137

Cattaneo, F. 1999, in Astrophys. Space Sci. Libr. 239, Motions in the Solar Atmosphere, ed. A. Hanslmeier \& M. Messerotti (Dordrecht: Kluwer), 119 Centeno, R., et al. 2007, ApJ, 666, L137

de Wijn, A. G., Rutten, R. J., Haverkamp, E. M. W. P., \& Sütterlin, P. 2005, A\&A, 441,1183

de Wijn, A. G., et al. 2008, ApJ, 684, 1469

Domínguez Cerdeña, I., Kneer, F., \& Sánchez Almeida, J. 2003, ApJ, 582, L55

Domínguez Cerdeña, I., Sánchez Almeida, J., \& Kneer, F. 2006, ApJ, 636, 496

Faurobert-Scholl, M. 1993, A\&A, 268, 765

Foukal, P., \& Milano, L. 2001, Geophys. Res. Lett., 28, 883

Goodman, M. L. 2004, A\&A, 424, 691

Harvey, J. W., Branston, D., Henney, C. J., \& Keller, C. U. 2007, ApJ, 659, L177

Hoge, E. R. 1947, PASP, 59, 109

Jendersie, S., \& Peter, H. 2006, A\&A, 460, 901

Keller, C. U., Schüssler, M., Vögler, A., \& Zakharov, V. 2004, ApJ, 607, L59

Lin, H., \& Rimmele, T. 1999, ApJ, 514, 448

Lites, B. W., et al. 2008, ApJ, 672, 1237

Martin, S. F. 1988, Sol. Phys., 117, 243

Martínez González, M. J., \& Bellot Rubio, L. R. 2009, ApJ, 700, 1391

Muller, R. 2000, in Allen's Astrophysical Quantities, ed. A. N. Cox (4th ed.; New York: Springer), 364

Orozco Suárez, D., et al. 2007, ApJ, 670, L61

Petrovay, K., \& Szakaly, G. 1993, A\&A, 274, 543

Pietarila Graham, J., Cameron, R., \& Schuessler, M. 2010, ApJ, submitted (arXiv:1002.2750v1)

Sánchez Almeida, J. 2004, in ASP Conf. Ser. 325, The Solar-B Mission and the Forefront of Solar Physics, ed. T. Sakurai \& T. Sekii (San Francisco, CA: ASP), 115

Sánchez Almeida, J., Asensio Ramos, A., Trujillo Bueno, J., \& Cernicharo, J. 2001, ApJ, 555, 978

Sánchez Almeida, J., Emonet, T., \& Cattaneo, F. 2003, in ASP Conf. Ser. 307, Solar Polarization 3, ed. J. Trujillo-Bueno \& J. Sánchez Almeida (San Francisco, CA: ASP), 293

Sánchez Almeida, J., \& Lites, B. W. 2000, ApJ, 532, 1215

Sánchez Almeida, J., Márquez, I., Bonet, J. A., Domínguez Cerdeña, I., \& Muller, R. 2004, ApJ, 609, L91

Sánchez Almeida, J., et al. 2007, A\&A, 475, 1101

Scharmer, G. B., Gudiksen, B. V., Kiselman, D., Löfdahl, M. G., \& Rouppe van der Voort, L. H. M. 2002, Nature, 420, 151

Schrijver, C. J., \& Title, A. M. 2003, ApJ, 597, L165

Schüssler, M. 1986, in Small Scale Magnetic Flux Concentrations in the Solar Photosphere, ed. W. Deinzer, M. Knölker, \& H. H. Voigt (Göttingen: Vandenhoeck \& Ruprecht), 103

Solanki, S. K. 1993, Space Sci. Rev., 63, 1

Spruit, H. C. 1977, Sol. Phys., 55, 3

Strous, L. 1994, PhD thesis, Utrecht Univ.

Title, A. M., \& Berger, T. E. 1996, ApJ, 463, 797

Trujillo Bueno, J., Shchukina, N. G., \& Asensio Ramos, A. 2004, Nature, 430, 326

van Ballegooijen, A. A., et al. 1998, ApJ, 509, 435

van Noort, M., Rouppe van der Voort, L., \& Löfdahl, M. G. 2005, Sol. Phys., 228,191

Viticchié, B., Sánchez Almeida, J., Del Moro, D., \& Berrilli, F. 2010, ApJ, submitted

Vögler, A., \& Schüssler, M. 2007, A\&A, 465, L43

Vögler, A., et al. 2005, A\&A, 429, 335

Wang, J., Wang, H., Tang, F., Lee, J. W., \& Zirin, H. 1995, Sol. Phys., 160, 277 